\title{
Hacia un derecho internacional privado europeo de sucesiones: la unificación de las normas de competencia*
}

\author{
Nerea Magallón Elósegui \\ Universidad de Deusto**
}

\begin{abstract}
Sumario: I. Intentos armonizadores del Derecho de sucesiones a nivel internacional. - II. El Libro Verde de Sucesiones y testamentos. III. La determinación del órgano jurisdiccional competente en el Reglamento 650/2012.-IV. Los criterios atributivos de la competencia en el Reglamento 650/2012. 1. Competencias generales. a) La residencia como criterio de competencia judicial. b) ¿Fórum non conveniens o prórroga de la competencia? 2. Las competencias subsidiarias o residuales. 3. El foro de necesidad. - V. La competencia limitada al lugar de situación de los bienes inmuebles en el Reglamento 650/2012. - VI. Conclusiones.
\end{abstract}

Resumen: El día 27 de julio de 2012 se publicó en el DOUE el Reglamento (UE) núm. 650/2012, sobre competencia judicial, ley aplicable y reconocimiento y ejecución en materia sucesoria. Se trata de un instrumento que desplazará la normativa autónoma de Derecho Internacional privado de los Estados miembros de la UE, introduciendo importantes modificaciones en nuestro ordenamiento. En este trabajo se realiza un sucinto análisis de la evolución que las normas de competencia han tenido a lo largo de todo el proceso normativo y de cómo han sido finalmente integradas en el nuevo Reglamento.

Palabras clave: Derecho internacional privado europeo, Derecho de sucesiones, normas de competencia.

Abstract: On July 27, 2012 was published in the OJUE Regulation $n^{\circ}$ 650/2012 EU on jurisdiction, applicable law, recognition and enforcement of decisions and acceptance and enforcement of authentic instruments in matters of succession. This instrument will displace autonomous Private International Law rules of the EU's Member States, introducing important modifications in our legal system. In this work, there is a succinct analysis of the evolution that the conflicts of

* Recibido el 24 de abril 2013, aceptado el 12 de junio de 2013.

** El presente trabajo se enmarca en la ejecución del Proyecto de Investigación subvencionado por el Ministerio de Ciencia e Innovación y el Feder [Ref. DER2010-15162 (subprograma JURI) y el Programa de consolidación y estructuración de unidades de investigación competitivas, subvencionado por la Xunta de Galicia y el Feder. 
jurisdictions rules have had along the whole normative process and of how they have been finally integrated in the new Regulation. dictions.

Keywords: European International private law, Successions, conflicts of juris-

\section{Intentos armonizadores del Derecho de sucesiones a nivel internacional}

Las Instituciones europeas no han sido las únicas en plantearse las ventajas que la armonización/unificación de las normas de Derecho internacional privado (DIPr.) puede suponer para solventar los problemas inherentes a las sucesiones de carácter transfronterizo ${ }^{1}$. Aunque con escasos resultados, los primeros intentos dirigidos a alcanzar la armonización del Derecho de sucesiones a nivel internacional se iniciaron a finales del s. XIX y principios del s. Xx. Tenemos que tener en cuenta que el Derecho de sucesiones es uno de los sectores del Derecho privado más arraigado a la identidad cultural de los Estados y las distintas concepciones existentes en la materia constituyen una muestra de la gran variedad de tradiciones jurídicas e ideológicas que se encuentran a nivel no sólo mundial si no también europeo ${ }^{2}$. El hecho de que en el Derecho de sucesiones se concentren cuestiones de Derecho de familia, de Derecho de bienes y de Derecho de obligaciones, incrementa las discrepancias existentes en los ordenamientos internos y los problemas que pueden surgir como consecuencia de esta pluralidad en las sucesiones transnacionales y, de un modo paralelo, las dificultades para lograr su armonización ${ }^{3}$.

La Conferencia de La Haya fue la primera en plantearse la posibilidad de abordar la creación de un instrumento de DIPr. en materia de sucesiones ${ }^{4}$. Ya en el año 1883 se elaboró en su seno un primer Proyecto relativo a los conflictos de leyes en materia de Derecho de sucesiones, testamentos

${ }^{1}$ Los problemas inherentes a una sucesión internacional son puestos de manifiesto en el Documento de trabajo de la Comisión que acompaña al Libro Verde de Sucesiones, SEC (2009)410 final, de 14.10.2009.

2 Vid. COLLINS, H., «European Private Law and the Cultural Identity of States», ERPL, 1995, pp. 353-365.

3 Cf. BATIFFOL, H., «Principes de droit international privé», $R$. des C., t-97, 1959, p. 440 ,

${ }^{4}$ Vid. OVERBECK, A.E. von., «Divers aspects de l'unification du Droit International Privé Spécialement en matière de successions», $R$. des $C$., 1996, V-II, t-104, pp. 533-630; STRAZNICKY, M., «Les Conférences de Droit International Privé depuis la fin de la guerre mondiale», R.des C, t-144, 1993-II, pp. 443-563. 
y donaciones ${ }^{5}$. Los trabajos iniciales aspiraban a ordenar conjuntamente las cuestiones relativas a Derecho aplicable, competencia y reconocimiento de resoluciones extranjeras en materia sucesoria ${ }^{6}$, sin embargo a la postre se conformaron con intentar acercar las reglas sobre conflictos de leyes ${ }^{7}$. Finalmente, en la Decimosexta Sesión de 1988 se adoptó el Convenio sobre ley aplicable a las sucesiones ${ }^{8}$ que se limitaba a regular los conflictos de leyes y dejaba a un lado el eventual acercamiento de las normas de competencia internacional. A pesar de la estrecha relación entre ambos sectores, la complejidad del tema (y el poco tiempo del curso $^{9}$ ) recomendaba ceñirse a los aspectos de ley aplicable y abordar más adelante las normas de competencia ${ }^{10}$. En el Derecho de sucesiones se puede decir que existe mayor consenso entre los ordenamientos de los Estados en lo que al ámbito de la competencia se refiere (en tanto prima el domicilio como criterio de atribución) que en el de la ley aplicable (donde la pugna entre unidad y el fraccionamiento aleja las posturas), no obstante, su omisión suponía una controvertida limitación a la universalidad del Convenio ${ }^{11}$. De esta manera la

5 Al que se sumaron sucesivas iniciativas durante los años 1904, 1925 y 1928; vid. LAINE, A., «Etude critique d'un Project de Convention concernent las solution des conflits de lois en matière de successions, de testaments et de donations à causa de mort», Rev. Cri, 1902 , pp. 227 y ss.

6 Vid. artículo 8 del Proyecto de Convenio de 1904 y arts. 8 y 9 del Proyecto de Convenio de 1925/1928.

7 A iniciativa de los suecos, tal y como destaca OVERBECK, A.E. von., en «Divers aspects...», loc.cit., p. 560; también STRAZNICKY, M., «Les Conférences...», loc.cit., pp. 30 y ss., y LAGARDE, P., «La nouvelle Convention de La Haye sur la loi applicable aux successions», Rev.cr. dr. int. pr., 1989, núm. 78, pp. 249-250; el tema se retomó en la Undécima y Duodécima Sesiones de la Conferencia de La Haya de 1968 y 1972.

${ }^{8} \mathrm{Si}$ bien no entró en vigor por carecer del número mínimo de ratificaciones requerido según su art. 28, vid. BORRÁS RODRIGUÉZ, A., «La Convention de La Haye de 1989 sur la loi applicable aux successions a causa de mort et l'Espagne», E. Pluribus Unum, Liber Amicorum Georges A. L. Droz, sur l'unification progressive du droit international privé, Martinus Nijhoff Publishers, The Hague, 1996; VAN LOON, H., «Towards a Convention on the Law Applicable to succession to the States of Deceased Persons», Hague Yearbook of International Law, vol. 1, 1988, p. 273.

9 Cf. VAN LOON, H., ibid., p. 276.

${ }_{10} \mathrm{Tal}$ y como se resalta en el «Rapport explicatif de M. Donovan W.M. Waters», Actes de Documents de la Seizième Session (1988), t-II, p. 534; y en «Conclusions of the Special Commission of November 1986 on the law applicable to decedents estates, drawn up by the Permanent Bureau, Preliminary Document No 4 of December 1986», en Actes et documents de la Seizième sesión (1988) t-II, p. 189.

${ }^{11}$ Cf., BORRÁS RODRIGUÉZ, A., «La Convention...», loc.cit., p. 9; a favor de tratar también las normas de competencia se pronunció el Sr. Lescaze de la Delegación Suiza y los profesores J. González Campos y la Prof. A. Borrás Rodríguez en nombre de la Delegación española, vid. «Procès-verbal No 1, Séance du lundi 3 octobre 1988(après-midi)», en et Documents de la Seizième sesión (1988) t-II, pp. 346 y ss; todo ello en ÁLVAREZ TORNÉ, M., Criterios de determinación de la competencia internacional en supuestos de sucesiones en el 
necesidad de elaborar un Convenio que se ocupara también de la competencia judicial en materia de sucesiones quedo latente ${ }^{12}$; y en la actualidad se aconseja seguir avanzando en el desarrollo de los trabajos sobre competencia, reconocimiento y ejecución de decisiones en este campo ${ }^{13}$.

Pero la Conferencia de La Haya no fue la única en proponer la eventual armonización del DIPr. de sucesiones. En la misma dirección es menester destacar las iniciativas procedentes de la Convención nórdica de 1934 (entre Suecia, Finlandia, Noruega y Dinamarca) y del Tratado de Benelux (entre Bélgica, Luxemburgo y Holanda) con objetivos parecidos pero igualmente con escasos resultados. Por su parte, desde las instituciones privadas sobresale la iniciativa del Grupo Europeo de DIPr. (GEDIP) que en su tercera reunión de trabajo llevada a cabo en Heidelberg, entre los días 30 de septiembre y 2 de octubre de 1993, elaboró un texto a modo de borrador de lo que podría ser un futuro Convenio sobre competencia judicial, reconocimiento y ejecución de resoluciones en materia de familia y sucesiones a nivel europeo ${ }^{14}$, que ha servido de inspiración a lo largo del proceso de elaboración del instrumento europeo.

El 27 de julio de 2012, tras un largo y arduo proceso normativo, por fin se publicó en el DOUE el Reglamento (UE) núm. 650/2012, de 4 de julio, del Parlamento Europeo y del Consejo relativo a la competencia, la ley aplicable, el reconocimiento y la ejecución de resoluciones, a la aceptación y la ejecución de los documentos públicos en materia de sucesiones mortis causa y a la creación de un certificado sucesorio europeo ${ }^{15}$.

\section{EI Libro Verde de sucesiones y testamentos: un ambicioso proyecto que incluye la unificación de las normas de competencia}

La producción de normas de Derecho institucional en cuestiones vinculadas al Derecho de familia y sucesiones se ha convertido en un objetivo que ha ido adquiriendo protagonismo de forma paralela a la necesidad de

ámbito de la UE, tesis 2010, Barcelona (inédita), pp. 264 y ss, y AZCARRAGA MONZONÍS, C., Sucesiones internacionales: determinación de la Ley aplicable, Tirant Monografías, 539, Valencia, 2008, p. 345.

12 Así lo ponían de manifiesto las Conclusiones y Recomendaciones adoptadas por el Consejo de Asuntos Generales y Política de la Conferencia de La Haya emitidas del 7 al 9 de abril de 2010.

${ }^{13}$ http://www. hcch.net/upload/wop/genaff2010concl_e.pdf

14 Vid. REDI (1991), 2, p. 596; REDI vol. XLV (1993), 2, pp. 637-642; el Informe explicativo redactado por el Prof. P. Lagarde puede verse en REDI., vol. XLVI (1994), 1, pp. 467474.

15 DOUE L 201/107, 27.07.2012. 
garantizar la libre circulación de personas y a hacer efectivo el principio de reconocimiento mutuo de resoluciones en el ámbito europeo ${ }^{16}$. Las Instituciones europeas llevan tiempo especulando sobre la unificación del DIPr. de sucesiones, y así se manifiesta con en el Plan de acción de Viena de 1998 en el que se concedía prioridad a la futura elaboración de un instrumento en esta materia ${ }^{17}$. Pero el empujón definitivo se originaba a través del Programa de La Haya ${ }^{18}$ que invitaba a la Comisión a elaborar un Libro Verde sobre sucesiones en el que se integraban, junto a los conflictos de leyes, las cuestiones de competencia, el reconocimiento mutuo y la ejecución de las resoluciones; amen de la creación de un certificado de herencia europeo. Poco después, tras la elaboración de un informe sobre el impacto que tendría un hipotético instrumento europeo en este área, complementado por un exhaustivo estudio realizado a encargo de la Comisión por el Instituto del Notariado alemán ${ }^{19}$, vio la luz el Libro Verde de Sucesiones y Testamentos (LVST) de 1 de marzo, de 2005. Al mismo tiempo se creaba un Grupo de Expertos (PRM III/IV) para que realizara un primer borrador de texto (Discussion Paper) ${ }^{20}$ que reflejaba sus primeros resultados en la Propuesta de Reglamento de 14 de octubre de 2009 (la Propuesta) ${ }^{21}$.

Desde el primer momento se apostó por la elaboración de un ambicioso instrumento en el que la unificación de las normas de conflicto de leyes debía combinarse con la de las normas sobre competencia y reconocimiento. En el LVST se aconsejaba abordar la unificación de las normas de compe-

${ }^{16}$ Proyecto de medidas para la aplicación del principio de reconocimiento mutuo de resoluciones judiciales en materia civil, C-12, DOCE de 15 de enero de 2001; vid. GÓNZALEZ BEILFUSS, C., «El Proyecto de medidas para la aplicación del principio de reconocimiento mutuo de las resoluciones judiciales en materia civil y mercantil», REDI, 2000-2, pp. 662-668.

17 Plan de Acción del Consejo y de la Comisión sobre la mejor manera de aplicar las disposiciones del Tratado de Ámsterdam relativas a la creación de un espacio de libertad, seguridad y justicia, de 3 de diciembre de 1998, publicado en el DOCE C19, de 23 de enero de 1999; vid. LAGARDE, P., «Vers un Règlement communautaire du Droit international Privé des régimes matrimoniaux et des successions» en Pacis Artes (obra Homenaje al Profesor Julio González Campos), vol-II, UAM/Eurolex, Madrid 2005, pp. 1686 y ss; RODRÍGUEZ BENOT, A., «Los avances de la normativa comunitaria en el reconocimiento de las resoluciones judiciales en otros sectores del Derecho de Familia: régimen económico matrimonial, parejas de hecho, alimentos y sucesiones», en La libre circulación de resoluciones judiciales en la Unión Europea. Actas del seminario, Universidad de Sevilla, Sevilla, 2005, pp. 159-183.

18 Programa de La Haya: diez prioridades para los cinco próximos años en el ámbito de la libertad, seguridad y justicia, aprobado por el Consejo Europeo de Basilea los días 4 y 5 de noviembre, C-53, DOUE C 53, de 3 de marzo de 2005.

$19 \mathrm{http} / / /$ ec.europa.eu/justice_home/doc_centre/civil/studies/doc_civil_studies_en.htm.

20 Documento de reflexión del Grupo de Expertos de 30 de junio de 2008 (Discussion Paper)

${ }^{21}$ COM (2009) 154 final, 14.10.2009. 
tencia ya fuera de manera paralela o con posterioridad a la de las normas de conflicto ${ }^{22}$. La interrelación entre las normas de conflicto y los criterios atributivos de competencia en este ámbito se pone continuamente de manifiesto $^{23}$. De hecho, las enormes diferencias existentes en los ordenamientos de los Estados miembros desde el punto de vista tanto sustantivo como conflictual, la diversidad reinante a la hora de utilizar un criterio de competencia u otro, así como las especificidades procesales propias del proceso sucesorio que dependiendo del Estado en el que se desarrolle involucra a autoridades administrativas, judiciales u otro tipo de profesionales como los notarios, lo convierte en un campo especialmente favorable tanto para la producción de conflictos negativos y positivos de competencia como para el forum shopping. Con todo, las partes involucradas en un proceso sucesorio transnacional se van a ver desbordadas por la incertidumbre y la inseguridad jurídica que caracteriza a este tipo de procesos de carácter internacional; y para afrontar tales inconvenientes se consideraba imprescindible abordar una unificación del DIPr. de los Estados miembros a un triple nivel. En este trabajo nos vamos a centrar en las cuestiones vinculadas a los criterios de competencia, realizando un recorrido por la trayectoria que han seguido las disposiciones de competencia general y de competencias especiales a lo largo del proceso de elaboración del Reglamento europeo.

\section{La determinación del órgano jurisdiccional competente en el Reglamento 650/2012}

Tratándose de Derecho de sucesiones en el que muchas de las cuestiones del proceso sucesorio se tramitan por autoridades no judiciales y de manera no contenciosa lo primero que nos planteábamos ante la apremiante aprobación de un instrumento europeo era la conveniencia de aplicar sus normas de competencia a autoridades no judiciales. De hecho es una de las preguntas que se trazó en el LVST ${ }^{24}$, en tanto era una de las cuestiones en las que se reflejaban mayores divergencias procedentes de los ordenamientos autónomos europeos. Desde el punto de vista sustantivo el tipo de auto-

${ }^{22}$ El LVST en primer lugar se refiere a la unificación conflictual y luego a la competencial, mientras en la Propuesta se ordena al revés.

${ }^{23}$ Incluso más que en otras materias, vid. FONT I SEGURA, A., «Valoración de las respuestas al Libro Verde sobre sucesiones y testamentos relativas a la competencia judicial», en VIÑAS, R. y GARRIGA, G. (coords.), Perspectivas del Derecho sucesorio, Marcial Pons, Madrid, 2009, pp. 60-81, p. 67.

${ }^{24}$ Apartado 3.3. Se plantea la aplicación de la norma de competencia judicial a otras autoridades y la posibilidad de que determinados trámites sean llevados a cabo por las autoridades de un Estado distinto al designado por la norma principal. 
ridad y el papel que desempeñan en el desarrollo del proceso sucesorio las autoridades judiciales y no judiciales varía mucho de un Estado a otro. Así mientras en el ordenamiento español el Notario se ocupa de la mayor parte de los trámites sucesorios y, salvo excepciones, el Juez únicamente participa en los procedimientos contenciosos; en Alemania el Juez tiene mayor protagonismo y en el Reino Unido se desconoce la figura notarial.

También se vislumbran enormes diferencias a la hora atribuir la competencia a las autoridades judiciales y no judiciales en los supuestos internacionales; si bien la mayoría de los Estados coinciden en que no aplican las mismas normas para determinar la competencia de unos y de otros órganos sino que la competencia de las autoridades no judiciales se establece en función de leyes especificas, normalmente correspondientes al domicilio del causante ${ }^{25}$.

En el proceso de elaboración del Reglamento europeo en materia sucesoria la preocupación por este tema se fue incrementando según se iba avanzando en la consolidación del texto definitivo en una clara tendencia a integrar todo tipo de autoridades, pero únicamente cuando ejercieran funciones jurisdiccionales. Las respuestas al LVST pusieron de manifiesto que las discrepancias se hallaban fundamentalmente en torno a las múltiples autoridades que se encargan del tratamiento sucesorio en cada Estado Miembro, de modo que lo que se propuso fue diferenciarlas en virtud de su capacidad o no para ejercer funciones judiciales en este $\mathrm{campo}^{26}$. Ello ha supuesto que en el desarrollo del Reglamento se terminara desvinculando la cuestión del concepto de autoridad del reparto de funciones para conocer un proceso sucesorio (que dependerá de cada ordenamiento interno) y se supeditara al alcance de la función que tales autoridades van a desempeñar. Así, en aras a esclarecer el ámbito de aplicación del Reglamento lo importante va a ser delimitar la función jurisdiccional que en cada Estado ejercen las autoridades competentes, pero seguirá correspondiendo a los Estados miembros distribuir la competencia.

\footnotetext{
25 Vid. JIMÉNEZ BLANCO, P., «Las declaraciones notariales de herederos en las sucesiones internacionales», AEDIPr, t. III, 2003, pp. 327-359, y LADROZ, G.A.L., «La activité notariale internationale», $R$. des $C$., t. 280, 1999, p. 73. En el documento de respuesta del GEDIP al Libro Verde se hace especial hincapié en este asunto y se pone en duda la potencial extensión de la norma a las autoridades no judiciales puesto que en muchos casos será la ley señalada en la esfera del Derecho aplicable la que se ocupará de establecer cómo se atribuye la competencia de este tipo de autoridades.

26 ÁlVAREZ TORNÉ, M., Criterios..., op. cit., p. 336, abogaba por modificar el término y referirse únicamente a «autoridades» cuando se trate de una competencia atribuida a autoridades judiciales y no judiciales y a «autoridades jurisdiccionales» para referirse únicamente a las autoridades estrictamente judiciales.
} 
En la Propuesta no hubo dudas a la hora de incluir a las autoridades no judiciales optando por una interpretación del término «órgano jurisdiccional» en sentido amplio que incluía a otras autoridades - en particular notarios y secretarios judiciales - cuando actúen por delegación ${ }^{27}$. Tampoco prescindió de ellas el Proyecto del GEDIP de 1993, ni el Discussion Paper del Grupo de Expertos que las integraba en su definición de autoridad a la par que recomendaba que se añadiera un anexo en el que cada Estado miembro precisara quiénes eran las autoridades que intervenían en la tramitación de una sucesión. Precisamente, el Capítulo II de la Propuesta se iniciaba con un artículo que se refería a las autoridades no judiciales solo que con reservas. Probablemente por ello fue posteriormente suprimido, y ya no se encuentra a partir del texto presentado, el 29 de junio, de $2011^{28}$. A tenor del entonces artículo 3 las normas de competencia del Reglamento se iban a aplicar a las autoridades no judiciales únicamente «en caso de necesidad», lo que producía cierta confusión sobre cuándo y hasta qué punto a ojos del Reglamento se equiparaba entre unas y otras autoridades ${ }^{29}$. Tras los debates y estudios que tienen lugar durante el segundo semestre de 2010 y primero de 2011 se establece la conveniencia de extender las normas de competencia también a otras autoridades y profesionales del Derecho cuando actúen no en caso de necesidad, si no por delegación de poder o bajo control de un órgano jurisdiccional. Suprimido el mencionado artículo y con el objetivo de delimitar qué tipo de autoridades pueden ser incluidas en este nuevo tenor en un principio se propone una remisión a los requisitos enumerados en la definición de «órganos jurisdiccionales» contenida en el Reglamento 4/2009 relativo a las obligaciones de alimentos.

En consideración con lo anterior el Reglamento desarrolla, por un lado, los Considerandos dedicados a esta cuestión y, por otro, amplia la definición de órgano jurisdiccional incluida en el artículo 3.2, insistiendo en varios puntos. Se recalca que el Reglamento no afectará a la organización interna actual de los Estados miembros, de modo que se respetarán las com-

27 En contra de extender la competencia a las autoridades no judiciales vid. HARRIS, J., «The Proposed EU regulation on succession and wills: prospects and challenges», Tru.L.I.2008, 22/4), p. 223.

${ }^{28}$ Elaborado conjuntamente por la Presidencia Húngara a la entrada de la Presidencia Polaca basado en las Orientaciones de la Presidencia al Coreper y al Consejo de 9 y 10 de junio de 2011 para allanar el camino e impulsar nuevos avances en el Reglamento europeo (11607/10 JUSTCIV 152 CODEC 968)

29 Vid. Max Plankt Institute for Comparative and International Law (MPI), «Comments on the European Commission's Proposal for a Regulation of the European Parliament and the Council on jurisdiction, applicable Law, recognition and enforcement of decisions and authentic instruments in matters of succession and the creation of European Certificate of Succession»; Version de 23 de Marzo, de 2010, en Rabels Zeitschrift 74, 2010, 3, p. 35. 
petencias de los órganos no judiciales - entre ellos de los notarios de los que se hace una mención expresa- en materia de sucesiones de cada Estado miembro. De esta forma se extienden las normas de competencia a aquellas autoridades no judiciales u órganos que ejerzan funciones judiciales «siempre que dichas autoridades y profesionales ofrezcan garantías en lo que respecta a su imparcialidad y al derecho de las partes a ser oídas», amén de que se sometan al Derecho del Estado miembro en el que actúen. Ello supone que sus decisiones deberán ser objeto de recurso o revisión ante autoridad judicial y tendrán fuerza y efectos similares a los de una autoridad judicial ${ }^{30}$. Por lo tanto, estas autoridades no se verán afectadas por el Capitulo II del Reglamento cuando no ejerzan funciones judiciales (como sucede en la mayoría de los países con los notarios), y para saber si su competencia se somete o no al Reglamento deberá verificarse su adecuación a lo estipulado en la definición del artículo $3.2^{31}$.

Para completar lo anterior, ante la dificultad de discernir realmente a qué autoridades se aplica en virtud de las marcadas definiciones, en el Reglamento se interpela a los Estados miembros a notificar a la Comisión las autoridades y profesionales a los que se refiere el artículo 3 con el objetivo de esclarecer definitivamente la cuestión. En el caso español entre las autoridades no estrictamente judiciales se está barajando incluir a los Secretarios judiciales y a los Notarios ${ }^{32}$ (estos últimos competentes para realizar las declaraciones de herederos ab intestato mediante acta de notoriedad art. 979 LEC y 209 bis Reglamento Notarial ${ }^{33}$ ). La consideración del Secretario Judicial como autoridad a efectos de aplicar el Reglamento es sin lugar a dudas adecuada en función de lo establecido en nuestro ordenamiento ${ }^{34}$. No obstante no vemos del todo clara la adecuación de la función notarial a las definiciones del texto europeo con lo que si así se hiciera sería oportuno plantearse realizar los pertinentes ajustes a nivel interno ${ }^{35}$.

30 Al igual que en el Reglamento 4/2009, de 18 de diciembre de 2008, relativo a la competencia, ley aplicable, el reconocimiento y la ejecución de las resoluciones y la cooperación en materia de obligaciones de alimentos.

31 Tal y como subraya en nota al pie la Nota de la Presidencia al Grupo de Trabajo de 29 de junio de 2011 (11870/11) para justificar la eliminación del artículo 3.

32 Agradezco la información a Isidoro Calvo Vidal, Consejero general de de la Unión Internacional del Notariado latino y Delegado para asuntos comunitarios del Consejo de los Notarios de la Unión Europea.

33 Artículo 979 redactado por Ley 10/1992, de 30 de abril, de Medidas Urgentes de Reforma Procesal. Reglamento Notarial: art. 209 bis, introducido por R.D. 1368/1992, de 13 de noviembre.

${ }^{34}$ En función del artículo 1 del Reglamento Orgánico del Cuerpo de Secretarios Judiciales, RD 1608/2005, de 30 de diciembre.

${ }^{35}$ Un ejemplo de ello en Res. DGRN de 18 de enero de 2005, CALVO VIDAL, I., «Nota», REDI; 2005-.2, pp. 1023-1033. 


\section{Los criterios atributivos de la competencia en el Reglamento 650/2012}

El Capitulo II del Reglamento regula la competencia judicial internacional mediante un sistema completo que entra en juego sin exigir la presencia de una conexión comunitaria y por el que se elimina el recurso subsidiario al sistema de competencia de los Estados miembros. El artículo 4 del Reglamento establece un foro de competencia general y unitario que al igual que el artículo 21, referido a la ley aplicable, señala como competente a la autoridad del Estado de la residencia habitual de causante en el momento del fallecimiento para conocer la totalidad de la sucesión. A continuación en aras a flexibilizar la competencia general se prevé una serie de competencias especiales entre las que se ha incluido una eventual prórroga de la competencia a favor del órgano jurisdiccional del Estado de la nacionalidad del causante, un forum non conveniens limitado, y una serie de competencias residuales que cubren aquellos supuestos en los que el causante reside en un tercer Estado cuando los bienes de la sucesión están situados en un Estado miembro. Para terminar, como veremos, se ha optado por prescindir del forum rei sitae.

\section{Las competencias generales}

a) La residencia como criterio de competencia judicial

La elección del criterio atributivo de competencia que con carácter general va a señalar al órgano jurisdiccional competente en materia sucesoria viene siendo desde hace tiempo una de las cuestiones más debatidas en el ámbito sucesorio. La diversidad de soluciones contenidas en los ordenamientos de los Estados miembros, aunque no es tan acusada como en lo que concierne a las normas de conflicto, incrementaba las dudas a la hora de elegir uno u otro criterio ${ }^{36}$. Sobre todo en tanto iba a suponer el desplazamiento de las normas autónomas de los Estados miembros.

Para determinar el criterio atributivo de competencia europeo más que por un método comparativo se ha optado por escoger un criterio autónomo

${ }^{36}$ Así el criterio predominante es el último domicilio del causante, por corresponder al lugar de apertura de la sucesión, aunque otros apelan al domicilio del demandado en los procesos contenciosos o al lugar de situación, sobre todo en lo que respecta a los bienes inmuebles. También hay Estados que acuden a la nacionalidad del causante o de las partes en el litigio y que prevén la posibilidad de elegir concedida al causante o a los herederos; vid. Étude de droit comparé sur les règles de conflits de juridiction et de conflits de lois relatives aux testaments et successions dans les Etats membres de l'Union Européenne, DNotI, 2002, pp. 33 y ss. 
en el que han de confluir los objetivos de integración y de cooperación propios de los instrumentos europeos con los valores subyacentes al ámbito material particular de que se trata. Todo ello sin olvidar la conveniencia de que los criterios atributivos de competencia concuerden con los criterios determinantes de la ley aplicable, pero sin renunciar a los caracteres propios del ámbito procesal en el que se desenvuelven ${ }^{37}$. Porque, aunque en materia sucesoria no se defienda un completo sometimiento de las normas de competencia a las normas de Derecho aplicable ${ }^{38}$ su concordancia favorecerá en gran medida el correcto desarrollo del proceso sucesorio ${ }^{39}$. Las dificultades inherentes a la aplicación del Derecho extranjero ratifican la conveniencia práctica de aplicar la ley del foro ${ }^{40}$.

A la hora de optar por un criterio u otro conviene equilibrar todos los principios que concurren bajo esta tarea. Desde el punto de vista de la integración europea las normas de competencia se deben a la construcción y afianzamiento de un espacio judicial común, y para cumplir tal propósito se orientarán a facilitar la cooperación entre autoridades y la eficacia en todos los Estados miembros de las resoluciones dictadas por autoridades de otro Estado miembro. De este modo se pretenden mitigar los problemas derivados de la diversidad resultante de la pluralidad de ordenamientos y asegurar el respeto a la tutela judicial efectiva mediante la defensa de los intereses y derechos de las partes con independencia de dónde se lleve a cabo el proceso sucesorio. Además, las normas de competencia deberán estar orientadas a promover la proximidad ${ }^{41}$ que garantice la accesibilidad de las partes a la autoridad competente y un grado de previsibilidad que les conceda la suficiente seguridad jurídica.

37 Vid. GONZALEZ CAMPOS, J., «Les liens entre la compétence judiciaire et la compétence législative en droit international privé», $R$. des $C$., t. 156, 1977, pp. 227-376 y BATIFFOL, H., «Observations sur les liens de la compétence judiciaire et de la compétence législative» en De conflictu legum: essays presented to Roeland Duco Kollewijn, Johannes Offerhaus, at their seventieht birthdays, NILR (ed), A. W., Sijthoff, Leiden, 1962, p. 66.

${ }^{38}$ Como veremos más adelante en el texto del Reglamento no se ha integrado un paralelismo automático forum/ius, pero se han incluido medidas que favorecen su afinidad.

39 Vid. DAVİ, A., «Riflessioni sul futuro diritto internazionale privato europeo delle successioni», Riv. Dr.int.pr, 2005, pp. 297-34, p. 307, y ÁLVAREZ TORNÉ, M., La autoridad competente en materia de sucesiones internacionales, Marcial Pons, Madrid, 2013, pp. 115 y ss.; FONT I SEGURA., A., «Valoración...», loc.cit., pp. 66-71.

40 Como es puesto de manifiesto por FORNER DELAYGUA, J.J., «Consideraciones acerca de la regulación de la competencia internacional de autoridades en un futuro Reglamento comunitario de DIPr relativo a las sucesiones por causa de muerte», en Perspectivas del derecho sucesorio en Europa, Marcial Pons, Madrid, 2009, pp. 83-109.

41 Vid. LAGARDE, P., «Le Principe de proximité dans le Droit International privé contemporain», R. des C., 1986-I, pp. 11-154. 
La residencia habitual ha sido el criterio elegido desde el primer momento en el Reglamento en materia sucesoria por ser el que mejor encarna todas estas necesidades (artículo 4). Por un lado porque es el criterio más utilizado por los ordenamientos nacionales de los Estados miembros, pero sobre todo porque suele ser el lugar donde se encuentran la mayor parte de los bienes que componen la sucesión, así como donde se hallan la mayoría de los interesados (potenciales beneficiarios y acreedores); con lo que implica un grado de vinculación entre el causante y el Estado que con frecuencia suele concordar con su centro de intereses - entendido como lugar donde tiene la mayoría de las relaciones familiares, sociales y patrimoniales $-{ }^{42}$. Estas cualidades también convierten a la residencia habitual en el criterio más adecuado al principio de proximidad que va a favorecer el acceso a la justicia a los interesados y a facilitar el correcto desarrollo del proceso. En concreto cuenta con la ventaja de que con carácter general implica una coincidencia entre forum y ius, con el consecuente beneficio, desde el punto de vista procesal y económico, que supone para las partes y para los órganos jurisdiccionales competentes la aplicación de su propia ley ${ }^{43}$.

Con el objetivo de desentrañar el concepto de residencia habitual en el Reglamento se han introducido ciertas pautas sobre el significado autónomo que se quiere dar a este concepto. En su Considerando 23 para determinar de un modo correcto cuál es la residencia habitual del causante se exhorta a las autoridades a hacer una evaluación de las circunstancias que han rodeado a su vida durante los años anteriores a su muerte. Para realizar esta labor las autoridades competentes deberán atender a la duración y condiciones de esa residencia (aunque no se estipulan plazos), así como a los motivos que han llevado al causante a ese lugar. Todo ello con el objetivo de reflejar la estrecha vinculación que garantice el cumplimiento de los objetivos del Reglamento y el porqué de la elección de este criterio.

Pero, además, en los supuestos en los que resulte difícil extraer de estos datos el lugar de la residencia habitual del causante, teniendo en cuenta la posibilidad cada vez más habitual de que el fallecido por motivos profesionales se hubiera trasladado a otro país pero mantuviera los vínculos personales con su país de origen, se puede convenir que éste último sigue siendo su verdadero lugar de residencia. En ese caso prevalecerán las circunstancias personales sobre las profesionales y se considera que el causante tiene su residencia habitual en el lugar donde se encuentra sus intereses sociales y familiares (Considerando 24). También se prevé la posibilidad de que el causante hubiera residido en varios países de un modo alternativo o que hu-

42 Entre otros, ÁLVAREZ TORNÉ, M., La autoridad..., op. cit., pp. 143-151.

${ }^{43}$ Con claridad FORNER DELAYGUA, J.J., «Consideraciones...», loc.cit., p. 84. 
biera viajado constantemente sin fijar su residencia de forma permanente, entonces se atenderá a su nacionalidad y a la localización de sus bienes como factores determinantes para fijar su residencia habitual, pero no se dispone cuál de las dos tiene más peso. Como vemos las pautas establecidas para discernir el concepto de residencia habitual adolecen de cierta vaguedad, lo que se puede traducir en altas dosis de incertidumbre para las partes. El Reglamento debería haber sido más específico y, para solventar las dudas que se pueden plantear, sobre todo en caso de segundas residencias, formular una serie de circunstancias concretas verificables a modo de test que ayudarían a despejar el significado autónomo de este concepto ${ }^{44}$.

\section{b) ¿Fórum non conveniens o prórroga de la competencia?}

A lo largo del proceso de elaboración del Reglamento la posibilidad de que sea el causante, sean las partes en un procedimiento sucesorio - normalmente los herederos - las que designen la jurisdicción competente para conocer parte o la totalidad de la sucesión se ha ido perfilando en torno a dos potenciales vías que, si bien a primera vista podían parecer diferentes, finalmente se han terminado fundiendo en una especie de híbrido especialmente diseñado para el ámbito sucesorio: la remisión a un tribunal mejor situado y la elección del foro.

En un principio la Propuesta de Reglamento, siguiendo el Discussion Paper del Grupo de Expertos incluyó un artículo inspirado en el artículo 15 del Reglamento de Bruselas II bis que mediante la incorporación de un forum non conveniens sui generis permitía la remisión del proceso de la autoridad competente (de acuerdo a las normas de competencia general) a los órganos jurisdiccionales de otro Estado miembro si consideraba que estaban mejor situados para conocer el asunto. De esta manera el artículo 5 de la Propuesta abría la puerta a la práctica de la transferencia de la competencia del tribunal de la residencia habitual del causante exclusivamente a favor del tribunal correspondiente al Estado de su nacionalidad y en aquellos supuestos en los que el causante, haciendo uso de la professio iuris hubiera elegido su ley nacional para regir su sucesión ${ }^{45}$. Bajo esta condición, con el objetivo principal de alinear forum y ius y de permitir al órgano jurisdiccio-

44 Tal y como sugería The Bar Council of England and Wales en su respuesta al LVST.

45 Art. 5 «Cuando el difunto hubiera designado la ley de un Estado miembro para someter a ella su sucesión de conformidad con lo dispuesto en el artículo 17, el órgano jurisdiccional que debería conocer del asunto conforme el artículo 4 podrá, a petición de una parte, y si considera que los órganos jurisdiccionales del Estado miembro están mejor situados para pronunciarse sobre la sucesión, inhibirse e invitar a las partes a plantear una demanda ante los órganos jurisdiccionales de dicho Estado miembro». 
nal competente aplicar su propia ley y así sortear los problemas y los costes inherentes a la aplicación de la ley extranjera, la Propuesta facultaba al órgano jurisdiccional que iba a conocer el asunto - siempre que lo estimara oportuno y a petición de una parte - a suspender el proceso e invitar a las partes a que acudieran a los tribunales del Estado miembro de origen del causante. Se trataba de acoger una solución más ajustada a aquellos supuestos en los que, habiendo residido el causante los últimos años de su vida en un Estado miembro distinto al de su nacionalidad, la jurisdicción de su lugar de residencia apenas presentara vínculos con la sucesión porque tanto sus bienes como las partes en el proceso se encontraran en el Estado de su nacionalidad. Existiendo tales vínculos cabía pensar que era la solución más adecuada, sobre todo en términos procesales.

De esta forma el artículo 5 de la Propuesta admitía una transferencia condicionada, que no iba a ser ni automática ni obligatoria ${ }^{46}$ y que dejaba al albur de la autoridad competente el ejercicio de la mencionada remisión. Con el objetivo de mitigar la incertidumbre y la consecuente inseguridad jurídica de las partes que ello podía causar, se establecieron unas orientaciones a tener en cuenta por la autoridad elegida a la hora de «considerar» tal transferencia. En ellas se apelaba a los intereses de las partes y a las particulares circunstancias que rodean la sucesión - como puede ser la residencia habitual de las partes o la situación de los bienes que componen el caudal hereditario - ${ }^{47}$. En el supuesto de que la autoridad competente estimara oportuno realizar la transferencia se invitaba a las partes a acudir a la jurisdicción señalada en un plazo determinado que, a su vez, debía declararse competente también en un plazo de tiempo más o menos amplio ${ }^{48}$. De lo contrario, al vencimiento de los plazos dictados el órgano jurisdiccional al que se sometió el asunto inicialmente seguiría conociendo el proceso. En definitiva, la transferencia dependía de la discreción del órgano jurisdiccional de la residencia habitual del causante y además, se limitaba únicamente a la concreta cuestión planteada ante dicho órgano; pudiendo suceder que se concediera en algunas cuestiones y que se denegara en otras ${ }^{49}$. Como consecuencia no se iba a conocer con certeza la jurisdicción competente ni se asegurarían plenos efectos a la autonomía de la voluntad del testador, po-

46 Tal y como se subrayaba en la Propuesta de 10 de octubre de 2009 comentada (COM 2009) 154 final.

47 Vid. Nota de la Presidencia al Grupo de Trabajo de 29 de junio de 2011 (11870/11).

${ }^{48}$ Primero se planteó que fueran plazo máximo de ocho semanas (Propuesta de Reglamento de 10 de octubre). Posteriormente se acorto a dos meses (Nota de la Presidencia al Grupo de Trabajo de 29 de junio de 2011). EL MPI propone que para evitar dilaciones se reduzca a dos semanas.

49 Vid. MPI, «Comments...», apdo. 100, p. 51. 
niendo en entredicho la deseada afinidad forum/ius y con ello los objetivos del Reglamento ${ }^{50}$.

No tardaron en surgir las dudas sobre si una remisión de estas características iba a ser suficiente o si por el contrario debería ser completada con la incorporación de cierta dosis de prorogatio fori para atender determinados supuestos ${ }^{51}$. Así, por ejemplo, si un holandés reside sus últimos diez años en España donde muere sin haber elegido la ley aplicable que va a regir su sucesión pero sin embargo todos sus bienes así como sus herederos se encuentran en los Países Bajos ¿es deseable que únicamente los tribunales españoles sean competentes para conocer todas las cuestiones de la sucesión $?^{52} \mathrm{O}$ en el caso de dos finlandeses que litigan en torno a la sucesión de un finlandés que muere residiendo en Lisboa ¿no tendría sentido que pudieran someter el litigio al tribunal de Helsinki? Y ello aunque se aplicara la ley de Lisboa ${ }^{53}$.

En cierto sentido la Propuesta se apartaba de la tendencia general seguida en otros instrumentos europeos que permiten la elección del foro ${ }^{54}$, y para remediarlo se terminó suprimiendo el artículo 5 y se modificó su contenido ${ }^{55}$.

50 Como dice FORNER DELAYGUA, J.J., «(...) en materia sucesoria la previsibilidad acerca de la ley aplicable es una extensión de la autonomía material que permite al testador disponer de los bienes; como esta autonomía corresponde al causante no son adecuados criterios de competencia internacional que no tomen en cuenta que un valor material del Derecho sucesorio es la autonomía material de quien no vive para defender y litigar la aplicación de la ley prevista», en «consideraciones...», p. 85; en efecto la falta de la autonomía de la voluntad del causante desde el punto de vista de la competencia convierte la elección de la ley aplicable en menos efectiva y previsible y el causante no sabrá con exactitud cuál será el Estado que aplicará la ley elegida; en este sentido LEIN, E., «Les compétences spéciales dans la Proposition de Réglement sur les Successions», en Bonomi/Schmid, Successions internationales, Réflexions autour du futur règlement européen et de son impact pour la Suisse, Zurich 2010, p. 92

51 Vid. GAUDEMET TALLON, H., «Les règles de compétence dans la proposition de règlement communautaire sur les successions», en Perspectives du droit des successions européennes et internationales : Étude de la Proposition de Règlement du 14 octobre 2009, Defrénois, Paris, 2010, pp. 121-134, p. 124; MPI, «Comments...», loc.cit., apdos. 97-111; LEIN, E., «Les compétences...», loc.cit., p. 92; BONOMI, A., «Prime consideración sulla porposta di Regolamento sulle successioni», Riv. Dir.int.pr.,N 4-2011 p. 899; BUHIGUES, J.L., «Desarrollo del espacio europeo de justicia: hacia el nuevo DI privado de sucesiones en la UE», Cursos de Derecho Internacional Y relaciones Internacionales de Vitoria-Gazteiz, 2008, p. 352.

52 Ejemplo puesto por GAUDEMET TALLON, H., «Les règles...», loc.cit., p. 124

53 Ejemplo tomado del Informe del Instituto notarial alemán.

${ }^{54}$ Art. 12 del Reglamento de Bruselas II Bis; Art. 23 Reglamento de Bruselas I o el art. 4 del Reglamento sobre obligaciones alimenticias; también permite la Ley de Derecho internacional privado suiza, art. 87 (2).

55 Acuerdo general sobre el texto articulado de la Presidencia al Consejo de 12 de diciembre de 2011, 18475/11. 
En lugar del artículo 5 el Reglamento introduce varias disposiciones que configuran un modelo que se aleja del Reglamento Bruselas II bis. El nuevo modelo se inicia con otro artículo 5 que sustituye la remisión a los órganos jurisdiccionales mejor situados por una elección limitada del foro. De este modo se introduce en el texto la posibilidad de que las partes interesadas acuerden acudir a los órganos jurisdiccionales cuya ley ha sido elegida por el causante para conocer sobre cualquier asunto relacionado con la sucesión. Como vemos se reconoce una prorogatio fori a todas las partes participes en la sucesión $^{56}$ limitada a los órganos jurisdiccionales del Estado de la nacionalidad del causante y condicionada a que éste haya hecho uso de la professio iuris a favor de la ley de su nacionalidad. En caso de que así fuera, el órgano jurisdiccional inicialmente competente deberá inhibirse y declinar su competencia a favor del órgano jurisdiccional de la nacionalidad del causante.

Con esta nueva disposición se asegura en mayor medida la concomitancia entre forum y ius y se reduce la eventual discrecionalidad del órgano jurisdiccional de la última residencia permitiendo que las partes acudan a litigar al tribunal de casa y por tanto apliquen su propia ley. Si se cumplen los requisitos estipulados la transferencia en este caso sí es automática y los órganos jurisdiccionales de la residencia deberán inhibirse y atribuir la competencia al órgano del Estado miembro cuya ley hay sido elegida.

Se trata de una decisión acertada aunque resulta excesivamente limitada. Estamos de acuerdo en reconocer una elección del foro limitada a determinados tribunales, puesto que de lo contrario se podrían producir abusos y vincular el supuesto a una jurisdicción con la que la sucesión no presentara ninguna conexión. Pero puede haber circunstancias en las que las partes tengan un interés legítimo en acudir a una autoridad distinta de aquella cuya ley rige la sucesión ${ }^{57}$. Se podría dar el caso de que el lugar de la última residencia del causante estuviera en un Estado A y de que con independencia de dónde se encuentren sus bienes, por ejemplo en el Estado C que a su vez corresponde al de su nacionalidad, todos sus herederos se encuentren en el Estado B. Si así fuera ¿No se debería posibilitar a los herederos a acudir a litigar al Estado B?; y si el testador reside en el Estado A y muere sin haber elegido la ley que va a regir su sucesión pero todos sus bienes y herederos

${ }^{56}$ En principio no se le conceda la facultad de elegir el tribunal al causante de la sucesión, tal y como planteaba la mayoría de la doctrina, vid. MPI «Comments...», loc. cit, apdos. 99-107, pp. 51-53; LEIN, E., «Les compétences...», loc.cit. p. 92; HARRIS, J., «The Proposed...», loc.cit., p. 220.

57 En el mismo sentido MPI, que propone la posibilidad de elegir también los tribunales del lugar de su anterior nacionalidad, de su anterior residencia, el lugar de la ley que rige su régimen económico matrimonial y donde se encuentran la mayoría de los bienes. Ahora bien todos ellos se tratan de tribunales de lugares a los que también extiende la professio iuris, asegurando de esta manera la coincidencia forum/ius. 
se encuentran en el Estado B, del que además es nacional ¿no tendría sentido que los herederos pudieran acudir al tribunal B?

El Considerando 27 del Reglamento zanja el tema pero resulta excesivamente rígido: las normas del presente Reglamento están concebidas para garantizar que la autoridad que sustancie la sucesión aplique la mayoría de los casos su propio Derecho, y los mecanismos que se establecen sólo podrán utilizarse cuando el difunto haya elegido la ley del Estado miembro del que ha sido nacional para regir su sucesión.

Por su parte el artículo 5 faculta a las partes a realizar un acuerdo sobre cualquier cuestión de la sucesión de forma similar a como establece el artículo 23 del Reglamento de Bruselas I. La pregunta es si mediante dicho acuerdo se puede llegar a otorgar competencia al nuevo órgano jurisdiccional para conocer sobre la totalidad de las cuestiones inherentes a la sucesión o si simplemente podrá conocer de la cuestión concreta sobre la que se elaboró el acuerdo; y en ese caso si su decisión será vinculante únicamente para las partes que lo realizaron. Debemos tener en cuenta que los acuerdos de elección del foro no deberían interferir en los intereses legítimos de terceras personas y que, sin embargo, muchas de las decisiones realizadas en el ámbito sucesorio son de carácter erga omnes con lo que podrían llegar a perjudicarles ${ }^{58}$. En previsión de esta posibilidad se ha incluido el artículo 9 que establece que, si a través de la comparecencia se pone de manifiesto que no todas las partes han sido parte del acuerdo de elección, el órgano jurisdiccional elegido deberá inhibirse cuando se impugne su competencia y en ese caso la competencia recaerá sobre el órgano competente de acuerdo a las reglas generales. Ahora bien, si la parte en cuestión que no hubiera formado parte del acuerdo comparece y no impugna su competencia el primer órgano jurisdiccional continuará con el proceso ${ }^{59}$.

Como podemos observar, el Reglamento en una fuerte apuesta por establecer la unidad del foro - a excepción de las competencias residualesno comprende la posibilidad de acudir a una autoridad distinta a la de la residencia o nacionalidad del testador ${ }^{60}$, y además, tal posibilidad en todo caso dependerá de la autonomía de la voluntad del testador. Lo que si se plantea es que aunque las partes no hayan pactado el órgano jurisdiccional al que someter el litigio puede ser igualmente conveniente en función de las circunstancias del caso acudir al de la nacionalidad del causante cuando se haya elegido su ley para regir la sucesión. Para ello el artículo 6 rescata el

58 Así lo resalta el MPI «Comments...», loc. cit., apdo. 11, p. 7.

59 Por el contrario el MPI en su apdo. 108, p. 53, planteaba la posibilidad de que ese acuerdo solo fuera vinculante para las partes que hubieran elegido a ese tribunal. .

${ }^{60}$ Como veremos mas adelante también suprime la posibilidad de acudir al foro de situación de los bienes inmuebles. 
forum non conveniens limitado que contenía la Propuesta y conviene que, cuando la ley elegida por el causante sea la de un Estado miembro, el órgano jurisdiccional inicialmente competente podrá declinar su competencia a instancia de una de las partes si considera que dicho órgano jurisdiccional se encuentra mejor situado para conocer el supuesto. En definitiva, se ha optado por un modelo hibrido que resulta excesivamente farragoso.

\section{Las competencias subsidiarias o residuales}

En virtud de la regla de competencia general del Reglamento (arts. 4 y 5) cuando el causante se encuentre residiendo en un tercer Estado en el momento de su fallecimiento las autoridades de los Estados miembros no tendrán competencia para tramitar su sucesión. No obstante puede suceder que esa residencia sea meramente coyuntural y que el causante conserve importantes vínculos con un Estado miembro. De hecho puede darse la circunstancia de que los bienes que componen la sucesión sí se encuentren en un Estado miembro. En ese caso, el Reglamento, en aras a garantizar la tutela judicial efectiva de herederos y acreedores, contempla en su artículo 10 la posibilidad de recurrir a los órganos jurisdiccionales de dicho Estado miembro.

El LVST se refería a esta contingencia de un modo categórico cuando apuntaba que «no es deseable renunciar unilateralmente a la competencia de los Estados miembros», sobre todo, «mientras que otros criterios de vinculación descartados a nivel intracomunitario serían pertinentes para determinar unilateralmente la competencia de estos órganos jurisdiccionales en relación con la de los terceros Estados». Acompañaba tal manifestación con un ejemplo ilustrativo de los inconvenientes que podían ocasionarse en el supuesto de un nacional de un Estado miembro que fallece residiendo en un tercer Estado mientras que tanto sus bienes como sus herederos y acreedores se localizan en un Estado miembro. Si así fuera, aunque concurrieran vínculos lo suficientemente estrechos con un Estado miembro, el criterio comunitario designaría a los órganos de un tercer Estado como competentes para conocer la sucesión, y herederos y acreedores podrían tropezar con enormes dificultades a la hora de acceder a justicia; todo ello aderezado con la potencial concurrencia de normas nacionales exorbitantes ${ }^{61}$.

Para remediarlo los instrumentos europeos vienen incorporando un artículo de competencia residual que se ocupaba de dejar el tema en manos

${ }^{61}$ Tal y como ponen de manifiesto las Orientaciones de 18 de Mayo de 2010 de la Presidencia al Consejo y Coreper. 
de las legislaciones nacionales ${ }^{62}$; tal y como dispuso el artículo 6 de la Propuesta del GEDIP de $1993^{63}$. Pero las dificultades que puede suscitar tal remisión para encontrar una solución común ${ }^{64}$ ha entrañado un giro en la valoración de la utilización de este tipo de técnicas ${ }^{65}$ que se refleja en el LVST. En él se propone incorporar una norma dirigida a armonizar las competencias residuales a nivel europeo ${ }^{66}$ que goza de muy buena acogida ${ }^{67}$. De hecho en el proceso de elaboración del Reglamento, visto el beneplácito existente en torno a la conveniencia de integrar dicha norma, el debate que rodea a esta cuestión se centró únicamente en los criterios que iba a contener tal disposición, su orden y relación.

El punto de partida de esta iniciativa lo encontramos una vez más en el Discussión Paper del Grupo de Expertos, cuyo contenido al respecto, ligeramente matizado, pasó a formar parte de la Propuesta de Reglamento. En su art. 2.3 establecía que, aún cuando la residencia del causante en el momento de su fallecimiento no estuviera en un Estado miembro, podrían ser competentes la autoridades de un Estado miembro cuando alguno de los bienes que componen la sucesión se hallarán en un Estado miembro y siempre que concurriera alguna de las siguientes circunstancias: que el causante, heredero o legatario, u cualquier otra parte interesada tuvieran la nacionalidad de ese Estado, o en su defecto que el causante hubiera tenido su última

${ }^{62}$ Cf. Art. 4 del Reglamento de Bruselas I.

63 Art. 6 «When the deceased did not have his habitual residence in any contracting State at the time of his death the Courts of any contracting State shall have jurisdiction if they have jurisdiction according to their own law».

${ }^{64}$ Sobre la disparidad que presentan los Estados miembros en materia de competencia residual y sus consecuencias, vid estudio realizado por el Prof. Arnaud Nuyts por encargo de la Comisión Europea, de 3 de septiembre de 2007 sobre las normas de competencia residuales de los Estados miembros aplicables de acuerdo al art. 4 del Reglamento 44/2001: http:// ec.europa.eu/justice_home/doc_centre/civil/studies

65 Vid. Libro Verde sobre la legislación aplicable y la competencia en asuntos de divorcio, COM (2005) 82 final, 14.03.2005 (punto 3.5), así como la Propuesta de Reglamento por la que se modifica el Reglamento (CE) n. ${ }^{\circ} 2201 / 2003$ por lo que se refiere a la competencia y se introducen normas relativas a la ley aplicable en materia matrimonial de 17.07. 2006; también Libro Verde sobre la revisión del Reglamento (CE) n. ${ }^{\circ}$ 44/2001 relativo a la competencia judicial, el reconocimiento y la ejecución de resoluciones judiciales en materia civil y mercantil, COM (2009) 175 final, de 21.04.2009; todo ello en BORRÁS RODRÍGUEZ, A., «Competencias exclusivas y competencias residuales: de Bruselas II a Roma III», en Essays in honeur of Konstantinos K. Kerameus, vol. I, Ant. N. Sakkoulas Publishers / Bruylant, 2009, pp. 165-182.

66 BONOMI, A., «The opportunity and the modalities of the introduction of Erga Omnes EC rules on jurisdiction», en The External Dimension of EC Private International Law in Family and Succession Matters, VV.AA., MALATESTA-BARIATTI-POCAR, (edts), pp. 149-160.

${ }^{67}$ Tanto por las respuestas al Libro Verde como por el Grupo de expertos Derecho Civil PRM III/IV. 
residencia en mencionado Estado; o en su defecto que un heredero, legatario u otra parte interesada tuviera su residencia habitual en dicho Estado miembro; o en su defecto que el causante haya sometido por disposición testamentaria el conjunto de la sucesión a la competencia de las autoridades de un Estado miembro; o en su defecto que la demanda se refiera únicamente a esos bienes. Además, a modo de cierre, se añadía un segundo párrafo referido a la ley aplicable por el que cuando la autoridad de un Estado miembro fuera competente en función de lo dicho con anterioridad la ley del foro regirá la sucesión excluyendo la posibilidad de reenvío.

La Propuesta, aunque se basó en el texto presentado por el Grupo de Expertos, eliminaba alguno de los criterios atributivos de competencia y alteraba ligeramente su orden, enumerando de nuevo los criterios atributivos de competencia que iban a acompañar a la situación de un bien como potenciales criterios residuales ${ }^{68}$ y así ha quedado plasmado finalmente en el Reglamento. En primer lugar se sitúa la residencia habitual previa del difunto otorgándole prevalencia sobre la nacionalidad. Ahora bien, para que dicha residencia sea suficientemente significativa se añade que en el momento en el que se someta el asunto a la autoridad no deberán haber transcurrido más de cinco años de la misma. Por lo que se deduce del texto esos cinco años se consideran tiempo suficiente para que el causante ya no mantenga allí su centro de intereses o tenga vinculación suficiente con ese lugar, así que transcurrido ese plazo la residencia previa dejará de ser relevante a efectos de señalar la autoridad competente. La lógica seguida parece basarse en que el transcurrir del tiempo comporta el consecuente desarraigo y, por tanto, el cambio de centro de intereses del de cuius. No obstante lo relevante a efectos de descubrir la suficiente vinculación con un lugar determinado no debería ser el tiempo que haya pasado desde que el causante tuvo dicha residencia sino cuánto tiempo estuvo residiendo en ese lugar. Es decir, bien podría suceder que la residencia previa hubiera tenido una duración que no le haya dado tiempo al causante a crear arraigo alguno. En previsión de estos casos hubiera sido más adecuado vincular el plazo al cómputo de la residencia y no al tiempo que ha pasado desde que residió en ese lugar. De esta manera cualquier residencia previa parece ser suficiente (aunque luego

${ }^{68}$ Art. 6 «Aún en el supuesto que el causante no tuviera su residencia habitual en el momento del fallecimiento en un Estado miembro, serán competentes los órganos jurisdiccionales de uno de ellos si alguno de los bienes estuvieran situados en dicho Estado miembro y si: a) el difunto hubiera tenido su anterior residencia habitual en dicho Estado miembro durante un periodo no inferior a cinco años antes de someter el asunto al órganos jurisdiccional o; b) el difunto poseyera la nacionalidad de dicho Estado en el momento de su fallecimiento o; c) un heredero o legatario tuviera su residencia habitual en dicho Estado o; d) la demandase refiriera únicamente a esos bienes». 
se matiza levemente en los Considerandos) siempre que allí se sitúe alguno de los bienes que componen la sucesión, con lo que al final la razón de peso se traslada a la ubicación del bien vaciándose el resto de contenido. Por el contrario podría suceder que aunque la residencia hubiera sido superior a ese plazo, por ejemplo de diez años, la vinculación subsistiera con independencia de que el causante llevare residiendo en otro Estado más de cinco años desde el momento en el que se acude al órgano jurisdiccional o se abre el proceso sucesorio; sobre todo si se dan las circunstancias de que en ese lugar se encuentran parte de los bienes que componen la herencia.

En segundo lugar, el artículo 6 de la Propuesta se refería al Estado de la nacionalidad del causante en el momento de su fallecimiento. La nacionalidad en un Estado miembro como potencial foro residual pasaba a situarse en segundo lugar y a aplicarse en defecto de que el causante hubiera tenido su residencia habitual previa en otro Estado miembro. Las ventajas inherentes a la residencia desalojaban momentáneamente a la nacionalidad que, a partir del Texto del Consejo de 12 de diciembre de 2011, volvía a situarse en primer lugar donde finalmente se ha asentado. La pugna entre ambos conceptos se podía haber zanjado como se proponía en los comentarios a la Propuesta del Max Planck Institut (MPI) que abogaba por que se convirtieran en foros alternativos separados por un simple «o» ante la dificultad de constatar las virtudes de la residencia en determinados $\operatorname{casos}^{69}$.

En el Discussion Paper del Grupo de Expertos también se incluía como posible foro de competencia la nacionalidad de alguno de los herederos, legatarios o de cualquier parte interesada, pero la escasa relación de este criterio con el centro de intereses del causante y la multiplicación de foros que comporta juega en su contra y finalmente no se ha incorporó ni en la Propuesta ni en el Reglamento aprobado. Lo mismo sucede con el criterio previsto en tercer lugar: la competencia del Estado en que uno de los herederos o legatarios tuviera su residencia habitual; en tanto su introducción podía incrementar los conflictos positivos de competencia y, cuando herederos, legatarios o interesados, residieran en diferentes Estados miembros, señalar a tantas autoridades competentes como participes en la herencia ${ }^{70}$.

69 Justifica esta opción a través del ejemplo de una mujer alemana que reside en su casa de vacaciones de España durante unos años antes de irse a vivir a Estados Unidos con su hija, donde muere dos años después. En este caso la competencia en función del artículo 6 será de los Tribunales españoles mientras que pueden haber también buenas (o incluso mejores) razones para que lo sean los tribunales alemanes y no los españoles, MPI «Comments...»loc. cit., p. 28.

70 En el mismo sentido, BONOMI, A., «Prime considerazioni sulla proposta di regolamento sulle successioni», Rivista di diritto internazionale privato et processuale, N 4-2010, pp. 875-914, p. 908; en contra de esta posibilidad, HARRIS, J., «The Proposed...», loc. cit., p. 223. 
Para terminar, cabe mencionar como potencial foro residual el Estado del lugar de presentación de la demanda, siempre que esta verse únicamente sobre los bienes situados en dicho Estado. Este foro previsto en la Propuesta se transformó a partir del Texto de la Presidencia, de 29 de junio, de 2011, en un segundo apartado del artículo 6 (artículo 10 del Reglamento), que dota de mayor flexibilidad a las normas de competencia residuales cuando los bienes se encuentren en terceros Estados. Sobre todo cuando los órganos jurisdiccionales de terceros Estados tengan competencia exclusiva y las decisiones otorgadas por los órganos jurisdiccionales en virtud del artículo 4 a 6 del Reglamento europeo corran el riesgo de no ser reconocidas ${ }^{71}$. Así, en el texto del Reglamento se suma al artículo 10 un segundo párrafo en el que se recoge la intención del apartado 6.d del Discussion Paper del Grupo de Expertos por el que cuando ningún órgano jurisdiccional de un Estado miembro sea competente en virtud de todo lo anterior, los órganos de los Estados miembros en los que se encuentren los bienes sucesorios serán no obstante competentes para pronunciarse sobre dichos bienes. De este modo la única conexión que se exige es la situación de los bienes y no es más que una competencia limitada a lo que concierne a dichos bienes ${ }^{72}$. Todo ello levemente matizado en el Considerando 30 del Reglamento en el que tampoco se aporta mayor claridad ${ }^{73}$.

\section{El foro de necesidad}

Aparte de las competencias residuales anteriormente mencionadas, de manera excepcional, para los supuestos en los que los órganos de un Estado miembro no resulten competentes pero tampoco lo sea ningún tercer Estado y, por tanto, concurra un conflicto negativo de competencia, así como para cubrir los supuestos en que siéndolo dicho órgano no se ocupe de realizar las oportunas actuaciones y se produzca una situación de indefensión y denegación de justicia a las partes, se ha estimado conveniente incluir en el Reglamento la competencia subsidiaria de los órganos de un Estado miembro. Tal y como apuntaba la doctrina ${ }^{74}$ a tales efectos podía tomarse de modelo el Re-

71 Nota al pie del artículo 4 del texto de la Presidencia al Grupo de Trabajo, de 29 de junio de 2011, 1870/11.

72 Vid. LEIN, E., «Compétences... », loc. cit., pp. 85-86.

73 En el mismo sentido, ÁLVAREZ TORNÉ, M., La autoridad...op. cit., 178.

74 BONOMI, A., «Prime considerazioni...», loc. cit., p. 909, y MPI, «Comments...», loc. cit., apdo. 94, p. 49; al igual que el Libro Verde de revisión del Reglamento 44/2001, p. 4, COM (2009) 175 final, de 21.04.2009. 
glamento 4/2009 en materia de alimentos ${ }^{75}$ que en su artículo 7 incluye un forum necessitatis ${ }^{76}$.Y así se hizo. A partir de la Nota de la Presidencia al Grupo de Trabajo, de 29 de junio, de 2011 se agrega al texto de la Propuesta un artículo 6a basado en la redacción del Reglamento 4/2009 que ha permanecido en los sucesivos textos hasta convertirse en artículo 11 del Reglamento.

Lo primero que debemos subrayar es que se trata de un foro a utilizar únicamente «en casos excepcionales», por tanto cuando un proceso no pueda «razonablemente» desarrollarse o iniciarse en un tercer Estado. Sin embargo es difícil predecir cuáles serán esas situaciones excepcionales. En el Reglamento sobre obligaciones de alimentos el Considerando 16 apuntaba como posibles supuestos «cuando en el Estado tercero de que se trate resulte imposible el procedimiento, por ejemplo debido a una guerra civil, o cuando no quepa esperar razonablemente que el solicitante conduzca un procedimiento en dicho Estado», y en el Reglamento de sucesiones se incluye el Considerando 31 de igual contenido.

En segundo lugar es menester subrayar que el forum necessitatis únicamente podrá ejercerse si el litigio guarda un vínculo suficiente con el Estado miembro del órgano jurisdiccional requerido. Nos quedará por tanto discernir qué se considera como una vinculación suficiente. El MPI llamaba la atención sobre los problemas que puede comportar la amplitud de esta definición que invita a litigar sobre la competencia ${ }^{77}$. En el Reglamento sobre obligaciones de alimentos se establecía a modo de ejemplo la nacionalidad de una de las partes, lo que en nuestro caso podría trasladarse a la nacionalidad del causante. Ahora bien, el vínculo también podría derivarse de la situación de algunos de los bienes en un Estado miembro ${ }^{78}$. De lo contrario si ninguno de los bienes se localizara en un Estado miembro ni la residencia habitual del causante estuviera en territorio comunitario se podría

75 Reglamento (CE) núm. 4/2009, del Consejo, de 18 de diciembre de 2008, relativo a la competencia, la ley aplicable, el reconocimiento y la ejecución de las resoluciones y la cooperación en materia de obligaciones de alimento, DO núm. L7, de 10 de enero de 2009.

76 Artículo 7, «Cuando ningún órgano jurisdiccional de un Estado miembro sea competente con arreglo a los artículos 3, 4, 5 y 6, los órganos jurisdiccionales de un Estado miembro podrán, en casos excepcionales, conocer del litigio si un procedimiento no puede razonablemente introducirse o llevarse a cabo o resulta imposible en un Estado tercero con el cual el litigio tiene estrecha relación. El litigio debe guardar una conexión suficiente con el Estado miembro del órganos jurisdiccional que vaya a conocer de él».

77 Cf. MPI., «Comments...», loc. cit., p. 49.

78 En el mismo sentido ÁLVAREZ GONZÁLEZ, S., «El Reglamento 4/2009 sobre obligaciones alimenticias», Diario La Ley, n. ${ }^{\circ} 7230,2009$, p. 9; lo que por otro lado lo acercaría a las competencias residuales tal y como destaca LEIN, E., en «Compétences...», loc.cit., p. 86. 
intentar aplicar este artículo para soslayar la competencia de los terceros Estados de un modo arbitrario ${ }^{79}$.

\section{La competencia limitada al lugar de situación de los bienes inmuebles en el Reglamento 650/2012}

Aparentemente existía un consenso generalizado en torno a la aceptación de que los aspectos reales vinculados a los bienes inmuebles que componen el caudal relicto fueran competencia de las autoridades del lugar donde se encuentran tales bienes ${ }^{80}$. De hecho tras plantearse dicha posibilidad en el LVST, siguiendo el camino trazado inicialmente por el GEDIP y por el Discussion Paper del Grupo de Expertos, la Propuesta integraba un artículo 9 que, en función de la estrecha vinculación existente entre la dimensión sucesoria y la dimensión relativa a los derechos reales, otorgaba una competencia excepcional para conocer los aspectos reales ligados a la trasmisión de un bien a los órganos jurisdiccionales del territorio en el que se encuentre ${ }^{81}$. La superposición de las cuestiones sucesorias con las reales, sobre todo en el caso de los bienes inmuebles, dificultaba la completa eliminación del foro del Estado del lugar de situación del bien ${ }^{82}$. En ocasiones la transmisión (y administración) de la propiedad comporta la adopción de medidas en relación al traspaso del dominio o a la inscripción registral en función de documentos que muchas veces las autoridades extranjeras no son capaces de proporcionar, especialmente en lo que a la administración y liquidación de los bienes inmuebles respecta ${ }^{83}$. Para hacer frente a tales exigencias la Propuesta preveía una competencia excepcional de los tribunales del lugar de situación del inmueble que se diferenciaba de los sistemas es-

79 Así lo ha apuntado ÁLVAREZ TORNE, M., La autoridad... op. cit., pp. 181-182.

${ }^{80}$ Vid. FONT I SEGURA, A., «Valoraciones...», loc. cit., p. 79.

${ }^{81}$ Art. 9 «Cuando la ley del lugar del Estado miembro en que esté situado el bien requiera la intervención de sus órganos jurisdiccionales para adoptar medidas de derechos reales relativas a la transmisión de dicho bien, su inscripción o su transferencia en el registro, serán competentes para adoptar tales medidas los órganos jurisdiccionales de dicho Estado miembro»

${ }^{82}$ Así lo afirmaba LAGARDE, P., «Vers....», loc. cit., p. 1700.

${ }^{83}$ Sobre los problemas que inherentes a la adopción de medidas relacionadas con los aspectos de administración de los bienes de la sucesión y la necesidad de que se tramiten ante autoridades correspondientes al forum rei sitae, vid, ÁLVAREZ TORNÉ, M., Criterios..., op. cit., pp. 458-464; un buen ejemplo de ello son los países anglosajones en lo que a la administración de la herencia se refiere, Al respecto RODRÍGUEZ BENOT, A., «La acreditación de la calidad de administrador de una herencia internacional: el certificado europeo de heredero», en Perspectivas del Derecho sucesorio en Europa, VIÑAS, R. y GARRIGA, G. (coors), loc. cit., pp. 175-194; HARRIS, J., «The Proposed...», loc. cit., p. 221. 
cisionistas $^{84}$ en que la competencia se limitaría exclusivamente a los aspectos reales ligados a la transmisión de la propiedad de los bienes - de modo que al resto de los derechos sucesorios se les aplicaría la ley señalada por las normas de conflicto - y con ello se facilitaría su reconocimiento en otro Estado. Lo primero que había que hacer era discernir cuáles eran los aspectos reales de la sucesión.

En principio se hubieran podido incluir todas las medidas relativas a la emisión de certificados y al tratamiento de aspectos registrales, de trasmisión de la propiedad y administración de la herencia ${ }^{85}$, pero no hubo el suficiente consenso al respecto ${ }^{86}$, y además ello podría comportar la fragmentación de la competencia en tantos lugares como bienes inmuebles existan. Así las Orientaciones políticas de la Presidencia al Consejo en aras a impulsar la continuación del proceso normativo ${ }^{87}$ se dedicaron en gran parte a delimitar entre el Derecho sucesorio y el Derecho de propiedad a efectos de la aplicación del Reglamento con el objetivo de excluir «los pertinentes aspectos sujetos a la legislación en materia de derechos reales».

Finalmente a partir del texto reformado de la Propuesta de 12 de diciembre de 2011, en el Reglamento se suprime el artículo 9, y en el artículo 1.2.1), referido a las materias excluidas de su ámbito de aplicación, añade a la naturaleza de los derechos reales «cualquier inscripción en un Registro de derechos sobre propiedades inmuebles o muebles sujetos a inscripción, incluidos los requisitos legales para llevarla a cabo, y los efectos de la inscripción o de la omisión de la inscripción de tales derechos en un Registro». Esta supresión se acompaña de una extensa explicación por vía de Considerandos que establece que el Derecho del Estado miembro en el que se sitúe el Registro determinará tanto las condiciones legales como las condiciones formales y las autoridades que van a verificar la concurrencia de todos estos requisitos; y el certificado sucesorio se utilizará para probarlo. También se excluirán los efectos de la inscripción de los derechos en el registro que se regirán por la ley del lugar del Registro.

84 Vid. LEIN, E., «Competences...», loc. cit, p. 87.

85 Vid. Propuesta del GEDIP.

86 Vid. HARRIS, J., «The Proposed...», loc. cit., pp. 221-222, otra opción seria la de diseñar documentos uniformes y que los actos relacionados con la administración de la herencia y a la trasmisión de la propiedad para que reciban un tratamiento uniformado se recomienda la elaboración de formularios, tal y como proponía RODRÍGUEZ BENOT A., en su aportación al LVST.

${ }^{87}$ De 6 de junio de 2011. 


\section{Conclusiones}

Las Instituciones europeas han apostado por la elaboración de un ambicioso instrumento en un ámbito especialmente sensible en el que como reflejan los antecedentes internacionales no era fácil llegar a un consenso. La aprobación del Reglamento europeo en materia de sucesiones supone por primera vez la unificación de las normas de competencia en el ámbito sucesorio a nivel europeo y la iniciativa ha sido bien acogida. Pero el Reglamento ha dejado alguna cuestión sin resolver y otras que podrían mejorarse. Entre otras cosas nos resulta excesivamente forzada la inclusión de los notarios como autoridades afectadas por la norma. En nuestro ordenamiento si queremos que sean considerados como autoridad competente a efectos del Reglamento habría que hacer las oportunas modificaciones. Además se podría haber sido más concreto a la hora de definir el concepto de residencia habitual que sigue quedando al albur de la interpretación del TJUE. Por su parte, la incorporación de la prorrogatio fori resulta excesivamente restringida e integrada en un modelo de transferencia de la competencia con un contenido inextricable. Sin embargo la definitiva exclusión del forum rei sitae debe interpretarse como una apuesta acertada a favor de la eficacia de las decisiones y los actos emitidos por un Estado miembro en los demás Estados miembros. A pesar de lo farragoso que en ocasiones resulta el texto nos encontramos ante una verdadera revolución del Derecho de sucesiones que valoramos positivamente. 